\title{
53rd National Congress of the Italian Society of Clinical Biochemistry and Clinical Molecular Biology (SIBioC - Laboratory Medicine)
}

\author{
Virtual Edition \\ 11-13 October 2021 \\ President of the Congress \\ Laura Sciacovelli (Padova) \\ Scientific Committee \\ Giovanni Palladini (Pavia) \\ Giuseppe Lippi (Verona)
}

Ada Aita, Gaetano Bernardi, Graziella Bonetti, Duilio Brugnoni, Marco Cantù, Aldo Clerico,

Giorgio Da Rin, Maurizio D’Amora, Antonio D’Avolio, Diego Faggian, Massimo Gion,

Roberto Guerranti, Giovanni Lombardi, Fabio Manoni, Alessandra Melegari, Silvia Mengozzi, Benedetto Morelli, Claudio Ortolani, Andrea Padoan, Giovanni Palladini, Paola Pauri,

Enza Pavanello, Paola Pezzati, Simona Pichini, Pamela Pinzani, Erica Rampoldi, Giulia Sancesario, Laura Sciacovelli, Alessandro Terreni, Matteo Vidali

\section{Scientific Secretariat}

Ada Aita, Fiamma Balboni, Umberto Basile, Sergio Bernardini, Graziella Bonetti, Ettore Capoluongo, Ciriaco Carru, Aldo Clerico, Giorgio Da Rin, Maria Stella Graziani,

Roberto Guerranti, Giuseppe Lippi, Martina Montagnana, Benedetto Morelli, Gavino Napolitano, Andrea Padoan, Enza Pavanello, Erica Rampoldi, Giulia Sancesario, Laura Sciacovelli,

Alessandro Terreni, Tommaso Trenti, Martina Zaninotto

*) These abstracts have been reproduced directly from the material supplied by the authors, without editorial alteration by the staff of this Journal. Insufficiencies of preparation, grammar, spelling, style, syntax, and usage are the authors. 


\section{THE TRANSPORT OF BIOLOGICAL SAMPLES: EXPERIENCE OF THE CAMPANIA REGION}

\section{D’Amora}

Director of the Integrated Department of Laboratory Medicine Asl Napoli 1 Centro

The DCA n. 55 of 30.09.2010 established the "Reorganization Plan of the Hospital and Territorial Laboratory Network". With note prot. 0406752 of 07.09.2020 the "Laboratory Medicine Network” Working Group was set up for the revision of the aforementioned Plan. The GDL has developed a technical document for the subject of "Blood and Biological Materials Transport Mode" which represents the legislation with which Campania has intended to regulate all activities relating to the transport of biological samples for all the services detailed in the document and has defined, for each, the methods of organization to ensure correct management of the process in all its phases through the traceability of the path and in compliance with existing procedures and protocols (Decree n.219 of 23/06/2021). The document highlights how these activities are also necessary to guarantee the quality of performance and the safety of operators. Until now there was no dedicated rule in Campania. The document was prepared with reference to the international and national professional guidelines of reference and provides, among other things, that the AASSLL, the AAOO, the University ones, the IRCCS and the accredited private sector, where there are methods different from what is foreseen, must adapt within six months of its issue. The following topics are dealt with in the annex to the decree: 1. Introduction 2. Definitions 3. Purpose 4. Transport regulations 5. Operating methods 5.1 Packaging of biological samples 5.2 Storage and transport of biological samples/ General recommendations 5.3 Storage and transport of biological samples Biochemistry and Hematology/Coagulation 5.4 Storage and Transport of Toxicology samples 5.5 Storage and Transport of Pathological Anatomy samples 5.6 Storage and Transport of Microbiology and Virology samples 5.7 Storage and Transport of biological samples for all Genetic investigations 6. Procedure for transporting biological material inside a health facility 7. Transport Card 8. Spillage of biological fluids 9. Traceability 10. Levels of responsibility 11. Clinical Chemistry and Hematology exam table with storage times and methods for each test 12. Microbiology/Virology exam table with times and methods of conservation for individual you st 13 Bibliography. The recommendations contained in the document refer to each determination that must be performed with the aim of proceeding in the most reliable way so as not to compromise the accuracy of its measurement from the end of the sampling to the beginning of the analysis, considering the following elements: the temperature of transport; the possible need to separate the plasma/serum from the corpuscular part; the possible need to freeze the sample (after centrifugation of the relevant tube); the temporal distance from the sampling phase. Conclusion: The transport of biological samples is now a strategic issue. The movement of them is no longer a rare event but a frequent practice, both due to the tendency of public structures to centralization and of private ones to consortium. Even the hub and spoke model and the flourishing of sampling centers see the handling of samples as the fulcrum of the activities aimed at improving the service to citizens. At the Italian level there is a lack of univocal regulations for which various regions have regulated the matter and today we can also include Campania among these.

\section{TELEMEDICINE AND HEMOSTASIS-THROMBOSIS CENTRES}

\section{Barcellona}

Dipartimento di Scienze Mediche e Sanità Pubblica Università di Cagliari, SSD di Emostasi e Trombosi Azienda Ospedaliero - Universitaria di Cagliari

Nowadays, telemedicine has different clinical applications since it is used in almost all medical specialties. The COVID-19 pandemic has created not only major economic and social upheavals but also has an important impact on public health. In this emergency situation, the use of telemedicine has been rise aiming to mitigate the effects of COVID-19 on human health (1).

In Italy, the Hemostasis-Thrombosis Centres (HTCs) belong to the Federation of Centres for the diagnosis of thromboembolic disease and the Surveillance of Anticoagulant therapy (FCSA) and they offer the highest possible quality of assistance to patients treated with Vitamin K Antagonists (VKAs) or Direct Oral Anticoagulants (DOACs). 
Unlike DOACs, VKAs need a close monitoring through the Prothrombin Time (PT) expressed as International Normalized Ratio (INR). The test result allows doctors at HTCs to prescribe the correct dosage of VKAs to maintain the INR in the therapeutic range. This means that patients have to go to their HTC more often than patients treated with DOACs. Telemedicine has been implemented in the routine clinical practice at HTC many years ago (2) and actually, during the COVID-19 pandemic, this system is of significant aid in the management of this therapy allowing patients to perform the test at home or to self-manage their own therapy.

The system is organised as a centralised net-supported program with a server and PC stations in the HTC and workstations in the peripheral districts. Points-of-Care INR allow patients to easy perform the test on capillary blood and to quickly gain the INR result thus reducing the number of controls that patients would perform at the HCT (3). This aspect is of important value during COVID-19 pandemic since overcrowding should be avoided.

In general, self-management and self-testing have similar safety $(\mathrm{RR}=1.08,95 \% \mathrm{CI} 0.81-1.45, \mathrm{RR}=0.99,95 \% \mathrm{CI}$ $0.8-1.23$, respectively) than traditional monitoring as reported by the metanalysis of Sharma et al. (4). As regard the efficacy, the authors showed a less incidence of thromboembolism when self-management was used ( $R R=0.51,95 \%$ $\mathrm{CI} 0.37-0.69)$ with a trend vs. a significant reduction in all-cause mortality ( $\mathrm{RR}=0.68,95 \% \mathrm{CI} 0.46-1.01)$ while selftesting allowed to reach a $4.4 \%$ (95\% CI 1.7-7.18) increase in time in therapeutic range. In accordance with other economic models, the metanalysis also showed that self-monitoring is cost-effective.

Another advantage of the use of telemedicine in HCT is the patients' satisfaction. In our experience (5) $85 \%$ of the patients are satisfied with self-testing at home and the quality of life is improved in $87 \%$ of them. The cost of test strips was medium-high for $89 \%$ of the patients, and $75 \%$ of them stated that it was worth improving their quality of life. Telemedicine is usefull in managing patients on VKAs and, as suggested by the FCSA (6), it is a safe and efficacy system to guarantee an adequate medical assistance not only routinely but especially during pandemic. Finally, telemedicine could be used also for DOACs patients putting in place a system that may allow patients to attach the PDF file of their laboratory tests and to video-call the doctors at HTC.

\section{References}

1. Doraiswamy S, Abraham A, MD; Mamtani R, Cheema S. Use of Telehealth During the COVID-19 Pandemic: Scoping Review. J Med Internet Res 2020;22:e24087.

2. Barcellona D, Fenu L, Cornacchini S, Marongiu F. Telemedicine can improve the quality of oral anticoagulation using portable devices and self-testing at home. J Telemed Telecare 2013;19:298-301.

3. Sharma P, Scotland G, Cruickshank M, Tassie E, Fraser C, Christopher Burton C et al. Is self-monitoring an effective option for people receiving long-term vitamin $\mathrm{K}$ antagonist therapy? A systematic review and economic evaluation. BMJ Open 2015;5:e007758.

4. Barcellona D, Fenu L, Marongiu F. Point-of-care testing INR: an overview. Clin Chem Lab Med 2017;55:800-805.

5. Barcellona D, Mastino D, Marongiu F. Portable coagulometer for vitamin K antagonist monitoring: the patients' point of view. Patient Prefer Adherence 2018;12:1521-26.

6. Poli D, Tosetto A, Palareti G, Barcellona D, Ciampa A, Grandone E et al. Managing anticoagulation in the COVID-19 era between lockdown and reopening phases. Intern Emerg Med 2020;15:783-86.

\section{TELEMEDICINE, REMOTE CARE AND INTEGRATION WITH GENERAL MEDICINE}

\section{A. Scatena}

Diabetology Unit Director, San Donato Hospital - Arezzo, Local health Authorities South East Tuscany

In Tuscany since 2008 management of people with diabetes mellitus is proactive, in order to do early diagnosis and slow down the onset of the complications. At the beginning of this experience general practitioners took care of patients with low level of assistance and diabetologists of patients with high level of assistance (Expanded Chronic Care Model). In 2016, with the creation of Individual Assistance Plan (PAI), general practitioners shared the management of high level assistance patients with the specialists. In 2020 PAI was extended at all the chronic patients of each level of assistance and shared with the specialist. In this way all the patients since diagnosis could be evaluated by the specialist and benefit 
from the most appropriate therapy and management. In the same period in Tuscany was created the diagnostictherapeutic pathway for adult people with diabetes mellitus, which outlines the criteria and the modalities of the interactions between general practitioners and specialists for the first access and in case of modification of glicometabolic control and acute or chronic complications appearance.

After that Covid-19 pandemic contributed to telemedicine diffusion and emergency needing to share patients data in order to promptly review the PAI. Therefore in May 2021 Tuscan region hypothesize also a software house for data sharing and the use of rapid modalities like Point of Care (POCT) and teleconsultation.

In our center we have carried on a pilot project with teleconsultation aimed to implement the diagnostic-therapeutic pathway for adult people with diabetes mellitus and to improve the visits and therapy appropriateness. 65 patients were included in the project. Teleconsultation with the general practitioner was carried out to discuss clinical features, glicometabolic control and cardiovascular risk class. Modification of therapy was shared and the side effects appearance (7.7\%) managed and solved in teleconsultation as well as the follow-up planning.

\title{
HOSPITAL AND TERRITORY: PROXIMITY MEDICINE AND THE ROLE OF THE CLINICAL LABORATORY FOR MONITORING THE DIABETIC PATIENT
}

\section{A. Ognibene}

\author{
Dipartimento di Medicina di Laboratorio e Trasfusionale USL Toscana Sudest
}

The Tuscany regional law n. 20/2020, in the preamble, establishes to "guarantee the assisted person the use of an appropriate assistance path in relation to the ascertained needs for health education, preventive services, social assistance, diagnosis, treatment and rehabilitation, where this requires the "intervention of several professionals in coordinated, integrated and programmed forms, the care of the assisted person must take place on the basis of an Individual Care Plan (PAI), which takes into account all the aspects that affect the patient's health, from those strictly health care to social ones, and which provides for the intervention of several professionals in coordinated forms, such as a multiprofessional team". The clinical problem must be recognized and identified promptly in such a way as to require an overall care of the patient by a multi-professional and multidisciplinary team, for clinical framing and/or monitoring. It is necessary to create a permanent link between the professionals involved and in particular between the general practitioner treating the patient, the outpatient specialist and the Laboratory and Diagnostic Service, between which the exchange of information and opinions must be constant, timely and also through the possibilities offered by "Digital Medicine" such as "the teleconsult", to define the path. The advantages of the multi-professional and multidisciplinary team and of the second-level pathology/specialty outpatient clinic concern all the actors involved: the patient who performs the diagnostic investigations; the General Practitioner (GP) who sees guaranteed the possibility of obtaining an answer to the clinical question or the scheduled management of controls in a short time; and the specialist doctor who uses all the diagnostic potentials present in the hospital or in a multi-specialist outpatient facility, to formulate, in a short time, diagnoses that require multidisciplinary interventions. The clinical laboratory is part of the team as a determining factor both to clarify the clinical question but also to establish the terms and times of 2nd level specialist intervention. The patient with known diabetes accesses the II level of care, exclusively by being sent by the GP using the priority classes as already indicated for the first visit or through a scheduled visit. The use of priority classes for patients in the care of the GP can take place whenever the current situation constitutes a novelty/variation compared to the previous equilibrium, such as to require an assessment in a short time. Different laboratory parameters represent indicators that, introduced in the priority decision algorithm, can, if communicated in a timely manner, define the path and strategies of specialist therapy in the most appropriate way. In the reality of the USL TSE, a project has been developed that allows to communicate via SMS the results of the laboratory parameters, related to diabetes monitoring, to the patient and the GP when these vary significantly compared to previous controls or represent a clinical state that justifies an intervention by the Team. 


\title{
THE PRE-ANALYTICAL PHASE IN THE POCT MANAGEMENT OF TERRITORIAL DIAGNOSTIC NETWORKS
}

\author{
C. Bellini ${ }^{1}$, M. Fantacci ${ }^{2}$ \\ ${ }^{1}$ Clinical Chemistry Laboratory Analysis, Misericordia Hospital Grosseto, South East Tuscany USL, Italy \\ ${ }^{2}$ POCT Network Director, Valdichiana Amiata Siena-South Laboratory Analysis Director, South East Tuscany USL, Italy
}

In recent years, the management of territory-based diagnostic networks has taken on a major role, in order to guarantee an effective and efficient service covering the entire territory. Most recently the need for a territorial response to Covid-19 has further highlighted its centrality. In this context the use of point-of-care testing (POCT) represents a valuable diagnostic opportunity that responds to the need for timeliness and proximity for the management of territorial emergencies and urgencies and to support the health activities of zonal hospitals, first-aid points and remote areas. The area of the USL Toscana Sudest is an example of such complexity, both for the extension covering more than $11,000 \mathrm{~km}^{2}$, with a population of about 800,000 inhabitants (density $72 / \mathrm{km}^{2}$ ), and for its variety, with $60 \%$ of municipalities being mountainous or partially mountainous and 1 island municipality. For several years now an ISO 9001:2015 certified territorial POCT Network has been organised to support and integrate laboratory analysis activities, coordinated by the Laboratories, which guarantees a timely and quality diagnostic service close to the places of care and also at domiciliary.Technological and analytical advances in POCT equipment, together with careful monitoring of Quality Control (QC), have improved the analytical quality of results. However in order to produce an overall quality result and minimise errors that may jeopardise patient safety, the whole process must be addressed for POCT, as we are used to doing for laboratory results. In detail several pre-analytical issues that can cause many potentially harmful errors, also considering the rapid availability of results, are worth focusing. A risk-based approach to POCT management allows to analyse all steps, to set up monitoring indicators to verify their correctness and to implement corrective and preventive measures to decrease the incidence of errors [1]. Traceability from request to report and registration of all operators and devices involved (doctor, nurse, patient, request, sample, batch, instrument) facilitate the workflow management and enable verification of data consistency. Besides the selection of tests (often already preset for the emergency), the timing of their execution useful for the clinical need and the correct preparation of the patient (e.g. fasting), great attention is paid to the procedure for unique identification of the patient and of the biological sample. Also the collection of the sample in terms of type, volume, device and sampling method, and the eventual treatment or storage before analysis are carefully controlled with shared checklists and operating procedures, as well as other QC on the sample, such as for example the presence of bubbles, clots or haemolysis.

It is therefore essential to act on training and to manage the skills of all the operators involved both in the use of the equipment and in its control in presence and remotely. The multidisciplinary committee is crucial in all aspects of the POCT network from the choice of tests, sites and patients, to the supervision of training and competency, as well as in monitoring performances [2].

\section{References}

1. Brun M, Füzéry AK, Henschke B, Rozak K, Venner AA. Identifying sources of error and selecting quality indicators for point of care testing. Pract Lab Med. 2021 Mar 21;25:e00216. https://doi.org/10.1016/j.plabm.2021.e00216. PMID: 33869709; PMCID: PMC8042172.

2. Nichols JH, Alter D, Chen Y, Isbell TS, Jacobs E, Moore N, Shajani-Yi Z. AACC Guidance Document on Management of Point-of-Care Testing. J Appl Lab Med. 2020 Jul 1;5(4):762-787. https://doi.org/10.1093/jalm/jfaa059. PMID: 32496555. 


\section{THE PREANALYTICAL PHASE IN THE DECENTRALIZED TESTING: DIFFERENCES AND COMPETENCES}

\section{G. Bonetti}

Laboratory of Clinical Pathology, Esine, Brescia, Italy

Point-of-care testing (POCT) can be used in clinical setting such as hospitals and in areas where decentralized testing is requested (i.e. pharmacies, patient homes, healthcare practitioner's office, residences for elderly, blood donation centers, in ambulances, public utility services). ISO 22870:2016, ISO 15189 and Joint Commission indicate that all hospital based POCT must be supervised by the central laboratory; the laboratory director is responsible for standards of performance in all domains of POCT. Decentralized diagnostics are affected by several organizational, environmental, operational and technical challenges. According to CLSI POCT04 (1) nonhospital based POCT programs should have a POCT director who has to develop a process to ensure compliance and implementation of quality testing. The personel responsibilities in nonhospital-based POCT are the same as hospital-based ones. In Italy there isn't a national rule for POCT but different local regional ones. Recently SIBioC WG on POCT produced a recommendation for the use of POCT in hospital setting (2). Laboratory errors may have serious consequences for patient health and outcome. The preanalytical phase is most vulnerable to errors (60-70\% of all laboratory errors). Preanalytical errors are quite frequent in decentralized diagnostic because it's usually performed by nonlaboratory personnel or by patients and because the increasing robustness and simplicity of currently available POCT systems can lead to the false perception that no risk or harm to the patient is possible. In CLSI POCTO7A the most common preexamination variables, potentially source of errors are listed (3). The most common preanalytical errors in decentralized diagnostics are related to patient preparation such as incorrect sampling time, to blood collection as patient identification, to sample handing such as inadequate sample mixing and tube filling, transport and to interferences such as hemolysis. Many constituents have a daily variation and the blood composition undergoes significant changes after food consumption. Sampling should preferably be done after an overnight fast from 7am and 9am and should always be done prior to the potentially interfering diagnostic and therapeutic treatments. If decentralized tests have to be performed at different times it is necessary to record sampling time and time of administration of any therapeutic treatments. For a proper patient identification it is recommended to use barcoding systems. Capillary specimen should be labeled with the patient's first and last name, identification number, date and time of specimen collection, and initials of the person collecting the specimen (4). The recommended order for microcollection is different from venous blood sampling: blood gases (they may be altered if sampling is delayed), EDTA tubes, tubes with other additives and serum tubes (4). Sample clots may cause spurious results, so it's necessary to invert the tubes gently several times. Any anticoagulated sample must be rejected if any detected clot are present. In capillary sampling when blood flow doesn't permit the right sample volume the massage and squeezing around the puncture site may cause falsely decreased concentrations of some analytes due to the dilution of the blood sample with tissue fluid and falsely increased concentration of potassium due to hemolysis. Hemolysis is the most frequent preanalytical error and can affect many assays. Spurious iperkaliemia in whole blood may be found using POCT such as blood gas devices if occult hemolysis is present and no HIL detection system is available.

Most preanalytical errors in decentralized diagnostics can't be detected but can be prevented by standard operating procedures and achieving proper personnel education not only in analytical but also pre and postanalytical phases.

\section{References}

1. CLSI. Essential Tool for Implementation and management of a Point-of-Care testing program. $3^{\text {rd }}$ ed. CLSI guideline POCTO4. Wayne, PA: Clinical and laboratory Standards Institute; 2016.

2. Rampoldi E, Patrucco G, Casati M, Morelli P, Carraro P per il Gruppo di Studio Point of Care Testing (POCT) Implementation and management of the point-of-care testing (POCT): essential indications. Biochim Clin 2021 (ahead of print), https://doi.org/10.19186/BC_2021.032.

3. CLSI. Quality Management: Approaches to reducing errors at the point of Care: Approved Guideline. CLSI guideline POCT07-A. Wayne, PA: Clinical and laboratory Standards Institute; 2010.

4. CLSI. Procedures and Devices for the Collection of Diagnostic Capillary Blood Specimens; Approved Standard-Sixth Edition. CLSI document GP42-A6. Wayne, PA: Clinical and Laboratory Standards Institute; 2008. 


\title{
THE ROLE OF LABORATORY MEDICINE IN THE MANAGEMENT OF MEDICAL DEVICES FOR SELF-MONITORING BLOOD GLUCOSE
}

\author{
M. Carta', G. Bonetti ${ }^{2}$ \\ ${ }^{1}$ UOC Medina di Laboratorio, Ospedale S. Bortolo, AULSS 8 Berica, Vicenza \\ ${ }^{2}$ Laboratorio Analisi ASST-Valcamonica, Esine
}

The self-monitoring of blood glucose is of utmost importance for diabetic patients: it allows patients to evaluate their individual response to the therapy and verify whether the glycemic objectives have been met. All of this must of course be based on the use of one data which accurately reflects the glycemia.

The guidelines recommend the use of a device for the self-measurement of glycemia (POCT) which passed the necessary tests of accuracy and precision and thus presents the CE mark. Some studies, however, have emphasized how a nonnegligible number of devices bears the CE mark without actually meeting the minimum criteria of accuracy requested (1).

Even the Italian guidelines (2) highlight the necessity that producing firms clearly declare the analytical features of their devices, particularly in terms of accuracy and precision. The hierarchy of the sources of information has the scientific studies of literature in the first place, with the declarations of producers only ranked second. Lacking explicit and comparable references or in the presence of diverging situations around the analytical performances, diabetic facilities may activate a local evaluation of themselves, with a periodic comparison of the accuracy of the devices being recommended anyway.

The evaluation of the accuracy of POCT systems is very discussed. ADA, FDA, CLSI and ISO 195-2013 (only to mention the most authoritative sources) report different targets. The Italian recommendations revolve around the norms ISO 1052013 which provide that at least $95 \%$ of the measures obtained with the glucometer differ of around $0.8 \mathrm{mmol} / \mathrm{L}$ with respect to the measurements obtained with the reference method for concentrations $<5.6 \mathrm{mmol} / \mathrm{L}$ and of around $15 \%$ for concentrations $>5.6 \mathrm{mmol} / \mathrm{L}$. Moreover, at least $99 \%$ of the measures must fall between the areas of error A and B displayed in the Clarke error grid (3). According to the FDA, on the other hand, the $95 \%$ of the values must not fall beyond $15 \%$ from the reference value and the $99 \%$ must be within $20 \%$.

In any case, the accuracy of glucometers is based on the comparison with a reference method, and it is thus necessary to utilize a correct reference method in laboratories.

Much has been discussed around possible variables that may alter the quality of a comparative study of this type (method used, type of capillary or venous sample, use of first or second drop for POCT measurement etc.) but what undoubtedly plays a major role is the use of procedures which can stop the in vitro glycolysis. Traditionally, sodium fluoride (NaF) is used to stop the glycolysis; this, however, is unable to contrast the glycolysis during the first hour of conservation of the sample. The use of inhibitors that associate the $\mathrm{NaF}$ with the citrate buffer thus provoking an early inhibition of the glycolysis has proved more effective in that sense.

This aspect has also been recently highlighted from the IFCC Working Group (5) and assessed in some studies.

The use of early glycolysis inhibitors (NaF plus citrate) has proved effective to stabilize samples even up to 15 days and this may allow their use even as control material to evaluate the analytical performances of the glucometers alternatively to the materials currently employed which are based on serum or plasma with an addition of glucose and which may present commutability problems.

\section{References}

1. Pleus S, Baumstark A, Jendrike N, et al. System accuracy evaluation of 18 CE-marked current-generation blood glucose monitoring systems based on EN ISO 15197:2015. BMJ Open Diabetes Res Care. 2020;8(1):e001067. doi:10.1136/bmjdrc-2019-001067

2. Carta, M., Testa, R. Raccomandazioni per l'autocontrollo della glicemia nel paziente diabetico: sinossi. Riv Ital Med Lab 10, 122-124 (2014). https://doi.org/10.1007/s13631-014-0050-6

3. International Organization for Standardization. In vitro diagnostic test systems-requirements for blood glucose monitoring systems for self testing in managing diabetes mellitus. ISO 15197:2013 
4. US Food and Drug Administration. Self Monitoring Blood Glucose Test Systems for Over The Counter Use. Guidance for Industry and Food and Drug Administration Staff. Available at https://www.fda.gov/regulatory-information/search-fda-guidance-documents/selfmonitoring-blood-glucose-test-systems-over-counter-use Document issued September 29, 2020, last accessed June 2021)

5. IFCC Working Group GMECC. How should glucose meters be evaluated for critical care. Available at https://www.ifcc.org/ifcc-educationdivision/emd-committees/c-poct/wg-gmecc/ (accessed June 2021)

\section{POST-ANALYTICAL PHASE IN DECENTRALIZED TESTING}

\section{Giavarina}

\section{Medicina di Laboratorio, AULSS 8 Berica, Vicenza}

As with tests in central laboratories, decentralized analyzes show risks of error in the post-analytical phase. Trying to outline some elements of this problem, it is possible to identify at least three critical elements with different peculiarities, with respect to "centralized" analyzes validation, reporting and, last but not least, clinical interpretation of the result.

We can discuss these items with respect to at least three macro areas: the skills of the personnel dedicated to POCT analysis, the available technologies and methodologies, the standardization of procedures.

The validation of the result is the decision to consider an analytical result "valid", reliable, to make decisions. Results from central laboratory are generally considered valid a priori. In the POCT setting, validation is responsibility of personnel performing the test, usually "non-laboratory" people who do not have the training that laboratory professionals do.

Validation of a result must also take into account at least the correctness of the pre-analytical and analytical phases, the devices functionality, as well as the verification of quality through the control systems. Controlling panic and delta check ranges adds further complexity. We can combat these risks of error with continuous training, such as in the pre-analytical and analytical phases; using updated technologies, which allow self-checks, warning systems, blocking of results in case of noncompliance with quality specifications, etc.; using software for self-validation and decision support. We are near the start of Artificial Intelligence and utilizing big data to prove competency of operators, to prove that meters were giving reliable results, close to the other meters in the hospital and to maintain quality control in devices used outside of the hospital and operated by nurses, emergency medical technicians, and others not laboratorians [1].

Numerous studies have shown that reporting can be a critical element, when the execution of the tests is very far from the control of the central laboratory. The example of carrying out tests at pharmacies is emblematic: for some tests, the analytical quality is now sufficient, but there is "an urgent need to adopt recommended decisional levels and reference ranges updated according to the more recently published clinical guidelines [2]. The incorrect laboratory report is the most relevant issue for the post-analytical POCT phase, and specific quality indicators specific quality indicators could be very useful or even mandatory soon [3].

Specific competence counts in the interpretation of the results. For some tests, such as blood gas tests or thromboelastometry, the skills of specialists who have POCT systems at their points of care are likely to be high, often the highest in the health care system. However, there is evidence for others tests and other clinical contexts (monitoring tests [4] or management of critical results [5]) the situation is very variable and sometimes worrying. The training of operators and the assessment of skills must therefore also concern, and with particular attention, the clinical significance of the results and the actions to be taken in the event of critical or unusual results.

\section{References}

1. Miller JA. https://www.aacc.org/cln/articles/2021/april/artificial-intelligence-is-poised-to-transform-point-of-care-testing (July, 2021)

2. Zaninotto M, Miolo G, Guiotto A, Marton S, Plebani M. Quality performance of laboratory testing in pharmacies: a collaborative evaluation. Clin Chem Lab Med 2016;54:1745-1751.

3. Brun M, Füzéry AK, Henschke B, Rozak K, Venner AA. Identifying sources of error and selecting quality indicators for point of care testing. Pract Lab Med 2021;25:e00216. https://doi.org/10.1016/j.plabm.2021.e00216 
4. Choi S, Choi SJ, Jeon BR, Lee YW, Oh J, Lee YK. What We Should Consider in Point of Care Blood Glucose Test; Current Quality Management Status of a Single Institution. Medicina (Kaunas). 2021 Mar 4;57(3):238. https://doi.org/10.3390/medicina57030238. PMID: 33806620; PMCID: PMC8001912.

5. Nichols JH, Alter D, Chen Y, Isbell TS, Jacobs E, Moore N, Shajani-Yi Z. AACC Guidance Document on Management of Point-of-Care Testing. J Appl Lab Med 2020 1;5:762-787.

\section{hs-cTnl: POINT OF CARE NETWORKS AND CLINICAL RISK}

\section{Mele}

Giomi

Unitaà operativa: Laboratorio Analisi Cliniche Struttura:ICOT Città':Latina Mansione:Responsabile del Servizio Laboratorio Analisi Cliniche

ISO 15189 and ISO 22870 enforce the implementation of dashboards, indicators, and patient risk management to continuously improve diagnostic outcome quality and TAT reduction. In acute coronary syndromes (ACS), not ST -segment elevation myocardial infarction guidelines (NSTEMI) we should determine how, troponin I - HS characteristic (CV contained within 10\%) and its 20\% delta value as significant variation in monitoring, they could invalidate the "negative predictive value" of an issued analytics data without an internal quality control that does not limit analytical imprecision, especially for concentration values near decision limits have. Our laboratory studied a 65-year-old patients who underwent synthetic treatment or prosthetic surgery for femoral fractures within $48 \mathrm{~h}$ of admission and had symptoms attributable to ischemic heart disease (dyspnea, chest pain, arrhythmia, and hypotension). Troponin dosage was measured in all these patients with third-party POCT quality controls; these controls were validated by dedicated software to reduce analytical variability and allow monitoring of high-risk patients directly in TIPO by cardiologists with protocol $0-1 \mathrm{~h}$; this also allowed the laboratory to measure, assess and reduce the risk of harm to the patient by IQCP system (Individualized Quality Control Plan) and monitoring integrated software as guidelines means to guarantee and protect above all the physician and the patient.

\section{THE RISE OF THE UBIQUITOUS LAB}

\section{G. Giannella}

Healthcare is the one of the largest success stories of our times. Technology is another of the largest success stories of our times. People expectations about healthcare + technology is at its absolute peak. We are in the middle of a health-tech secular change. This is for good. This is unstoppable. This is the best part of the story. Yet, healthcare spending is unsustainable in an aging world. Technology, as every tool, brings its risks. Global levels of assistance are outrageously unequal. Mental disorders are exponentially growing. That's the other part of the story. How will the lab of the future adapt to the entire story? Will it "just" be able to sustain the health-tech revolution, or will it also play a role in making global health systems more sustainable, equal, and safer?

The speaker of this panel will present his view on how "deeptech" (a synergetic combination of leading-edge technology which today assembles Internet of Things, Artificial Intelligence, Digital and Space) coupled with rising individual expectations for highly personalized and accessible healthcare, will redefine the boundaries of healthcare system and the concept itself of $l a b$.

Internet of Things will be the main key to acquire all the right data. Trillions of "sensors" supporting data gathering from multiple sources: humans, plants, things, places, food, animals, environment. Contextualized data gathering to acquire different data in different contexts, "actuators" instructing devices to do the right thing in the right moment, surveillance networks for enhanced threats.

Artificial Intelligence will be non-optional. With health-related knowledge doubling in months, AI will become a mandatory survival kit. Yet, it still will see things that human eye might miss. Or making correlations that are simply too difficult anyway else. Yes, it will be both defensive for professionals and offensive to diseases, if used in the right way, as every tool. 
Digital will influence behaviors, create communities, and redefine the patient-professional interaction. With big part of the clinical outcome depending on context and behaviors, Digital will help to "nudge" patients with personal, doable, life-enhancing tips. But Digital is also the "home of increased expectations". Patients are individuals, parents, children, workers, citizens, consumers, with ever-growing expectations on what and how can be done through a smartphone. Health won't make any exceptions.

Space and satellite technology will provide the communication background for all above, from remote surgery to distributed expert network, etc. But Space will also bring additional data coming from macroscopic data gathering, earth observation, context-related data and gravity-less phenomenon analysis.

Can the lab of the future stay immune from all above? Hard to believe. While exact predictions are useless, some trends are clearly visible and point to the raise of a next-generation ubiquitous lab. An "always-on" set of competences, data, technologies, uniquely positioned to close the fracture existing between everyday life/health of individuals and the infinitely smaller portion of its current clinical representation.

\section{POCT IN HAEMATOLOGY: TECHNOLOGY AND NEW OPPORTUNITIES}

\section{R. Pajola}

U.O.C. Laboratorio Analisi, ULSS 6 Euganea, Ospedali Riuniti Padova Sud “Madre Teresa di Calcutta”, Monselice (Pd)

The complete blood count (CBC) is one of the most requested tests, routinely performed in the central laboratory (LAB) by large haematological analysers, useful to diagnose many diseases and manage urgent clinical decisions such as transfusion or administration of chemotherapy and antibiotics.

In recent years, rapid technological improvements led to the spread of point of care testing (POCT), performed outside the LAB and capable of providing CBC in a few minutes, measuring haemoglobin ( $\mathrm{Hb})$, red blood cells (RBCs), white blood cells (WBCs) with their differential count of 3 or 5 populations, platelets (PLTs) and many other parameters. Hb remains the most common POCT in haematology, essential to exclude anaemia.

There are two types of technology: small benchtop analysers and portable devices. The former are smaller and fully automated versions of LAB's analysers and, although portable, are not suitable to use at patient's bedside. The latter, some of which use disposable cartridges, do not require start-up procedures, maintenance and calibrations.

The latest generation of portable devices combines advanced digital technology with innovative technologies of viscoelastic focusing and microfluidics and techniques, such as digital microscopy and computer vision, using near infrared spectroscopy and the absorption of light at multiple wavelengths, obtaining CBC results unthinkable until a few years ago.

POCTs' accuracy is influenced by several factors: sample collection, blood type (venous or capillary) and timing. Poor finger prick technique can provide misleading results, it was proved that capillary samples significantly underestimate PLTs overestimating $\mathrm{Hb}$ and WBCs, but differences have not clinical relevance when the samples are collected according to standardized procedures.

POCT devices can not differentiate normal cells from pathological ones (e.g., erythroblasts, blasts, etc.). The presence of large platelets can lead to inaccurate PLT counts compared to the impedance method used in LABs. As recommended by the guidelines, due to the inherent risk of preanalytical errors and the standard risk of error during analytical and postanalytical phases, threshold values must be established to repeat CBC in a LAB.

Another consideration is POCTs' cost, which is cumulatively higher than that of a LAB-performed CBC.

Literature suggests that POCTs are not yet the ideal tools to perform $\mathrm{CBC}$ for diagnostic purposes, but they are useful in urgent situations such as rapid monitoring of some parameters (e.g., WBCs and $\mathrm{Hb}$ ). Further studies are needed to confirm the promising results of POCTs and evaluate their performance even at low ranges and in pathological conditions.

\section{References}

1. Rampoldi E., Patrucco G., Casali M., Morelli B. Carraro P. per il GdS POCT. Principi per l'implementazione e la gestione del point-of-caretesting (POCT): indicazioni essenziali. Biochim Clin 2021; 45: 312-326

2. Briggs C., Kimber S., Green L. Where are we at with point-of-care testing in haematology?. BJH 2012; 158: 679-690.

3. Mooney C., Byrne M., Kapuya P. et al. Point of care testing in general haematology. BJH 2019; 187: 296-306. 


\section{PAST PRESIDENT ASSOCIAZIONE MEDICI DIABETOLOGI}

\section{N. Musacchio}

Milano

Machine learning algorithms have proven to be very effective in predicting the behavior of phenomena represented in biomedical data.

The most commonly used machine learning algorithms, such as artificial neural networks, produce so-called "black box" results, namely:

- a complex set of mathematical equations that cannot be interpreted by people who do not have in-depth mathematical skills;

- predictive models that do not provide any explanation on the motivation underlying the forecast.

When applying machine learning to data such as images, black box algorithms are not a problem, since the value of the model lies in its accuracy in detecting the presence of certain patterns, attributable, for example, to the presence of a tumor. However, IF THE PURPOSE IS TO OBTAIN A DEEPER UNDERSTANDING OF A SPECIFIC PROBLEM, IT IS FUNDAMENTAL TO KNOW WHY THE PREDICTIVE MODEL CLASSIFIED, FOR EXAMPLE, A PARTICULAR PATIENT AS BEING 'AT RISK' OR 'NOT AT RISK’ BY ELICITING THE CHARACTERISTICS ASSOCIATED WITH THIS CLASSIFICATION.

A specific ML technique, the Rulex "rule generation method", builds models described by a set of intelligible rules, thus allowing the extraction of important knowledge regarding the variables included in the analysis and their relationships with the outcomes of the phenomenon analyzed. "Clear box" artificial intelligence enables exciting scenarios for true augmented intelligence, in which humans and machines can create a synergy - always controlled by a human "domain expert" - for a step forward in improving the quality of care.

Last but not least, according to the GDPR, personal data must be processed transparently and all the UE citizens have the right to an explanation if a decision concerning them has been made with artificial intelligence algorithms: the AI 'clear box' works in compliance with European regulations.

\section{RISK MANAGEMENT FOR POINT-OF-CARE TESTING}

\section{J.H. Nichols}

PhD, DABCC, FAACC

Vanderbilt University Medical Center, Nashville, Tennessee, USA

Introduction: Point-of-care testing (POCT) is laboratory testing conducted close to the site of patient care. POCT is growing in popularity with manufacturers offering a wide menu of tests and devices where the operator can obtain a rapid test result with the potential to initiate faster patient care decisions. But POCT is not fool-proof, and any test can and will fail if operated under the wrong conditions.

Methods: Risk management is a process where laboratories can assess their weaknesses, implement a control plan to detect and prevent erroneous results, and monitor the effectiveness of their plans.

Results: The Clinical and Laboratory Standards Institute (CLSI) EP23-A: Quality Control Based on Risk Management provides guidance based on risk management for laboratories to develop quality control plans tailored to the particular combination of measuring system, laboratory setting, and clinical application of the test.

Discussion: This presentation will describe how laboratories can partner with manufacturers to conduct risk assessments and implement quality control plans in their laboratory and at the point-of-care. The advantages of utilizing a risk management approach to controlling laboratory errors will be emphasized along with the efficiencies gained from conducting a risk assessment and implementing a quality control plan. A revision of CLSI EP23-A is currently being drafted, and this presentation will preview a few of the updates that can be expected in the next version of the guidance document. 


\title{
EBLM: ITS VALUE AND ROLE IN DECENTRALIZED DIAGNOSTIC AND PROXIMITY MEDICINE
}

\author{
V. Pecoraro \\ Dipartimento integrato di Medicina di Laboratorio e Anatomia Patologica, Modena
}

Evidence based laboratory medicine (EBLM) focuses on the use of diagnostic tests to improve patient outcomes. Principles of EBLM, integrating the best research and the clinical expertise, could be applied also for point of care testing (POCT) (1). POC are tests conducted near the site of patient care, outside of the laboratory, usually performed by patients or clinical personnel not trained in laboratory medicine. POCT require small sample volumes, minimize pre-analytical errors, and reduce alterations of labile analytes. However, when used appropriately, could improve the patients outcomes by providing faster results and earlier therapeutic strategies (2). Instead, its over or incorrected use could leads a patient risk and potential increase of healthcare costs. We assessed, through a systematic review of the recent scientific literature, the accuracy of the POCT on troponin, procalcitonin, C-reactive protein, parathyroid hormone, INR and d-dimer, and evaluate the impact of faster results on patient management. Studies measuring PCT, PTH and d-dimer reported a limited impact on diagnostic decisions. Instead, studies on CRP claimed a significant reduction of antibiotic prescription. Several authors evaluated troponin and INR reporting faster decision-making without any improvement in clinical outcome. Faster results are often translated in better outcomes, without evidence to support this conclusion. So, it is important that the POCT practice is evidence-based looking for evidence of whether POCT confers any advantage in clinical decision making in different scenarios. In some settings, such as rural environment, a rapid availability of cardiac troponins or other analytes can help clinicians to rule out or rule in disease, without transfer patient in other center, avoiding unnecessary costs (3). Likewise, in Emergency Department, availability of more rapid results with POCT help clinicians to refer patients, but does not always translate into shorter stays (4). The satisfactory analytical performance, together with an excellent practicability, suggest that the POCT represents an important technological advance in patient care, but, the lack of evidence about the patients outcome invite healthcare workers to use it with judgement.

\section{References}

1. Price CP. Point of care testing. BMJ 2001; 322: 1285-1288

2. Pecoraro V, Banfi G, Germagnoli L, Trenti T. A systematic evaluation of immunoassay point-of-care testing to define impact on patients' outcomes. Ann Clin Biochem. 2017 Jul;54(4):420-431.

3. Herd GCE, Musaad SMA. Point-of-Care Testing in Rural and Remote Settings to Improve Access and Improve Outcomes: A Snapshot of the New Zealand Experience. Arch Pathol Lab Med. 2021 Mar 1;145(3):327-335

4. Alter DN. Point-of-Care Testing for the Emergency Department Patient: Quantity and Quality of the Available Evidence. Arch Pathol Lab Med. 2021 Mar 1;145(3):308-319

\section{EBLM AND BLOOD GAS ANALYSIS PARAMETERS: WHICH IS MANDATORY TO MEASURE}

\section{E. Rampoldi}

Coordinatrice del Gruppo di studio POCT per SIBIOC

Arterial and venous blood gas analysis reveals oxygenation and acid-base status of the body. The analysis usually includes: $\mathrm{pH}, \mathrm{PaO} 2, \mathrm{PaCO} 2$ and a wide range of different analytes, besides other several derivated (calculated) parameters, as HCO3-, BE, anion gap.

Arterial $\mathrm{pO} 2(\mathrm{PaO} 2)$ is the most important variable to assess the oxygenation status and can't be substituted by venous blood or capillary measures of $\mathrm{O} 2$ (1).

American Association for Respiratory Care (AARC) updated Recommendations on blood gas analysis (BGA) and hemoximetry (2). The clinical practice guideline is based on 237 clinical trials, 54 reviews, and 23 meta-analyses 
searched in MEDLINE, CINAHL, and Cochrane Library database. Hemoximetry is recommended to determine the impact of dyshemoglobins on oxygenation. Some calculated values may be in error, e.g. calculated SaO2 may overestimate oxyhemoglobin saturation in the presence of carboxyhemoglobin or methemoglobin. Moreover, the presence of high concentration of fetal hemoglobin may also be a problem if blood gas analyzer does not detect it, as instrument assumes hemoglobin to be of the adult type, and therefore the calculated blood gas oxygen saturation values are underestimated.

In critically ill patients many other analytes have been used to estimate the severity of disease and try to prognosticate morbidity and mortality. No measurements can encompass the complexity of a disorder, but lactic acid can approach that goal (3) Indeed lactic acidosis is the most frequent metabolic acidosis and many causes are reported for lactate increase, not only hypoxia: the higher the lactate concentration, the worse the outcome. The initial values have a prognostic significance, but serial measurements are more valuable for prognosis. Conductivity-based Hematocrit (Ht) estimations have limitations. Abnormal protein concentration will change plasma conductivity. Low protein concentration, resulting from dilution of blood with protein-free electrolyte solution during surgery, will result in erroneously low $\mathrm{Ht}$ value. Conductivity-measured hematocrit during and after surgery could produce inaccurate results when Ht are lower than $30 \%$, and, therefore, result in unnecessary red cell transfusions in some patients. In any situation, to correctly interpret BGA results history should be always considered: reasons for presentation, information concerning events, environment, trauma, medications, poisons, toxins and an accurate physical examination should be carefully collected.

\section{References}

1. Acute respiratory distress syndrone: the berlin definition, Ranieri MV et al. 2012 Jun 20; 307(23):2526-33. https://doi.org/10.1001/jama. 2012.5669

2. AARC Clinical Practice Guideline: Blood Gas Analysis and Hemoximetry: 2013 Michael D Davis, Brian K Walsh, Steve E Sittig, and Ruben D Restrepo Respir Care 2013; 58(10):1694-1703.

3. N Engl J Med Lactic Acidosis 2014; 371:2309-19. https://doi.org/10.1056/NEJMra1309483

4. Conductivity-based Hematocrit measurement during cardiopulmonary bypass. Steinfelder-Visccher J et al. 2007; J Monitoring and Computing, 27:7-12

\section{DECENTRALIZED COVID-19 DIAGNOSTICS: RISKS AND OPPORTUNITIES. RAPID SEROLOGICAL TESTING}

\section{G. Lippi}

Section of Clinical Biochemistry, University of Verona, Verona, Italy

The knowledge that has been garnered so far on severe acute respiratory syndrome coronavirus 2 (SARS-CoV-2) infection is that humoral immunity encompasses the generation of immunoglobulins of most classes against surface viral antigens, which mostly involve the spike protein, the nucleocapsid protein, but also envelope and membrane proteins. Since the spike protein is the anchor that the virus uses for penetrating the host cells through biding with its natural host cells receptors, it can be assumed that antibodies binding to spike protein of SARS-CoV-2, and especially to its receptor binding domain, would retain stronger neutralizing potency against the virus. Serological testing has been conventionally defined as a diagnostic procedure used for detecting an immune response against an infectious agent. This definition shall dissipate any residual doubt about the fact that this type of testing is not intended to replace the identification of viral RNA for diagnosing SARS-CoV-2 infection, but must be rather used for establishing if individuals have been infected by the virus and/or have developed an immune response. The diagnostic sensitivity stratified according to the assay methodology is highly variable. Recent evidence confirms better performance for chemiluminescent and ELISA anti-SARS-CoV-2 IgG immunoassays compared to lateral flow immunoassays, whose sensitivity remains far below $80 \%$. Additionally important drawbacks of rapid serological tests include the facts that the information provided by the companies is concerning because often lacks details, its quality is considerably variegated among 
different devices, several claims are vague, there is a lack of transparency along with the fact that human aspects are not been adequately addressed for purpose of alleviating the risk of inappropriately using the device. The risk of misinterpreting tests results by patients when rapid kits are used for self-diagnosis is another aspect that must be considered. Recent evidence was provided that over $2 \%$ of patients may misdiagnose themselves as being negative while the device generates positive results. This was mostly due to objective difficulties encountered by the patients in reading and interpreting the results of the strips. Important considerations for implementing point-of-care serology testing thus include (i) usage of well-validated tests, evaluated against a gold standard; (ii) performance characteristics - thus encompassing sensitivity, specificity, positive and negative predictive values or cross-reaction with other coronaviruses - shall be tested using serum samples collected from patients infected with SARS-CoV-2, with other respiratory viruses (including seasonal coronaviruses) and also from healthy controls; (iii) adequate training of healthcare workers is needed (iv) and, finally, (IV) provisions must be in place, encompassing the capture of testing data for individual patient records and surveillance purposes, and the participation to external quality assessment schemes, to systematically monitor the quality of this type of testing.

\section{WE ARE IN THE MIDST OF THE FOURTH INDUSTRIAL REVOLUTION}

\section{Notarangelo}

“Avv. Valentino Notarangelo, Data Protection Officer \& Legal Counsel nei settori banking e sanità”.

At the heart of society 4.0 there are data, which are the engine of everything and, through them, progress, economy and, today more than ever, health and scientific research move.

It is in these sectors that, lately, the greatest investments have been made in digital transformation aimed at exploiting -through data-all the new present and emerging technologies, from the Internet of Things (IoT) to Artificial Intelligence (AI). The exploitation of Big Data, in fact, constitutes the starting point and the indispensable resource for the development of innovative and precision medicine, providing scientific, organizational and infrastructural support to promote research and accelerate preclinical and clinical studies.

However, this development, having increased the number of subjects holding health-related data, the speed of transmission of such data and the quantity of information electronically stored (often not on national territory), has determined an exponential increase in the danger of data processing from the point of view of confidentiality and an increased possibility of damaging the dignity and fundamental freedoms of the individual. This has led to an increased sensitivity of the European legislator and, subsequently, of the national legislator, towards the protection of such data and related protections. In addition to the General Data Protection Regulation, which has revolutionized the way of conceiving the data economy, it is, in fact, being evaluated by European institutions the first draft of the Artificial Intelligence Act, which will be the real springboard for the massive and regulated use of algorithms, especially in healthcare.

To be precise, this last mentioned regulation will only define the limits to the use of algorithmic systems already widely in use. Proof of this is the recent intervention of the "Italian Drug Agency" (AIFA, Agenzia Italiana del Farmaco), ${ }^{1}$ which wanted to recall and emphasize all the regulations to be respected in order to submit and conduct trials using algorithms and Machine Learning techniques. AIFA, through this guide, has described some case studies, showing some workflows that represent the regulations impacted depending on the type of system used and paying particular attention to the compliance related to the treatment of data and the related profiles of cybersecurity.

The challenge in the use of health data with application of AI systems in healthcare and scientific research is, therefore, to transform the "usual" classification of a disease into a better clinical decision-making process, defining more precisely the characteristics and features that the individual pathology highlights in the case in question, and then obtaining - thanks to algorithms and machine learning processes - specific, personalized, possibly predictive therapy protocols.

1 AGENZIA ITALIANA DEL FARMACO (AIFA), Guidance on submitting an application for a Clinical Trial involving the use of Artificial Intelligence (AI) or Machine Learning (ML) systems, 24.05.2021 https://www.aifa.gov.it/. 


\title{
DRIVE THROUGH DIFESA: L'ITALIA DIVENTA AREA DI CRISI
}

\author{
C. Renzi \\ Policlinico Militare - Roma
}

Con l'avvio dell'Operazione IGEA, la Difesa ha fornito il suo supporto al Servizio Sanitario Nazionale per l'attività di screening del Coronavirus mettendo a disposizione della Nazione circa 200 postazioni distribuite su tutto il territorio nazionale. Ad oggi sono operativi circa 72 Drive-Through-Difesa. I contributi, forniti da ciascuna Forza Armata, sono diretti e coordinati fin dalla prima ora dal Comando Operativo di vertice Interforze (COI) per mezzo di una Sala Operativa dedicata, composta da personale interforze. In aggiunta al Policlinico Militare di Roma “Celio" sono stati inoltre messi a disposizione anche ulteriori 11 laboratori in tutta Italia e sono già stati eseguiti ben oltre 2,9 milioni di tamponi. Il concorso della Difesa nel contrasto al COVID 19 ha visto impegnati dal 23 ottobre 2020 circa 1.895 militari al giorno, per un totale di 434.107 giornate/uomo complessive. Con l'arrivo delle prime dosi di Vaccino, su richiesta della Struttura Commissariale, il Comando di Vertice Interforze della Difesa ha avviato l'Operazione EOS e, facendo tesoro dell'esperienza e delle specifiche competenze logistiche acquisite in questi anni di impegno nelle varie missioni nei diversi Teatri Internazionali, ha approntato e implementato un piano di distribuzione delle dosi mettendo a disposizione un dispositivo, prontamente adattabile in base alle esigenze, composto da 11 aerei, 73 elicotteri e 322 mezzi. Nel valutare alcune richieste pervenute dalle ASL, la Difesa ha successivamente convertito i Drive Through in "presidi per la somministrazione dei vaccini"(PVD), attività questa che si è aggiunta a quella già in atto, di stoccaggio dei vaccini presso l'aeroporto di Pratica di Mare e il successivo trasporto in tutte le regioni italiane. Ad oggi sono stati attivati sul territorio nazionale 30 Presidi Vaccinali della Difesa che supportano la Sanità Nazionale con le vaccinazioni a favore della popolazione civile, i quali hanno eseguito già oltre 473 mila vaccinazioni. Ad essi si aggiungono i 35 Presidi Vaccinali Mobili della Difesa con il compito di supportare la campagna vaccinale soprattutto in quelle località più difficili da raggiungere, che hanno eseguito oltre 53 mila vaccinazioni. E' stata avviata inoltre la campagna vaccinale a favore delle isole minori tra cui le Tremiti, le Eolie, Pantelleria, Ustica, le isole della regione Sardegna, l'isola di Capraia e del Giglio, per le quali sono state somministrate oltre 25 mila vaccinazioni. Preziosissimo il contributo del Policlinico Militare "Celio", uno dei tre Ospedali Militari di riferimento, che è stato riconvertito in tempi rapidissimi in Covid Hospital ed inserito nella rete nazionale anti Covid quale riferimento delle strutture sanitarie del Centro Italia, mettendo a disposizione 152 posti letto di cui 100 di degenza ordinaria e 52 di sub-intensiva/intensiva.

\section{LABORATORY MEDICINE WOULD MERIT A FURTHER SMALL REVOLUTION}

\section{F. Salvatore}

Professor of Human Biochemistry, Emeritus Dipartimento di Medicina Molecolare e Biotecnologie Mediche Università di Napoli Federico II

CEINGE-Biotecnologie Avanzate Founder and Principal Investigator Past (president and scientific coordinator) Member of Accademia Nazionale delle Scienze (detta dei XL) - Roma

In the last couple of decades, Laboratory Medicine has made giant steps forward in terms of innovative technology and has made major scientific breakthroughs in the medical field as a whole. Indeed, a plethora of both in vitro and in vivo assays and tests in biological fluids of the human hydrodynamic system are now available.

The importance, for clinical purposes, of novel metabolic processes and protein cross-talk mechanisms is being increasingly recognized. The increased survival period of sick, elderly people, plus the therapeutic aspects of precision medicine, in which the drugs selected resulted in a series of direct approaches to altered target molecules, have made it difficult to identify the most effective molecules to use as biomarkers in most of this population scenario.

In this optics, the clinical value of Laboratory Medicine has now reached about $70 \%$ of the most important measures used to diagnose diseases, but at a cost to the National Health System of many countries that reaches a maximum of $5 \%$ of the global health costs. Therefore, it seems that Laboratory Medicine does not need to increase further value in the contribution to the care of fragile individuals, and in people affected by chronic degenerative diseases. 
Notwithstanding all these premises, and the increase in Clinical Laboratory testing, which is, and will continue in the future to be an indispensable ally of medical care, the correct diagnosis of a single or of multiple diseases occurring in a single individual will benefit enormously from this Discipline, if some steps forward will be made. I believe that the enormous amount of knowledge now accumulating in the field of Laboratory Medicine will revolutionize, not only the medical care of people, but, in the various areas of the medical scenario, also the field of Laboratory Medicine Science itself and the practice deriving from it.

However, there is a grey, largely neglected area in humankind: although life-span has almost doubled in the last 150 years, I believe the time has come to look at the wellbeing of each individual during his/her lifespan, particularly because multimorbidity occurs during the lifetime of each individual. In other words, we should all begin to be mindful of our state of health as early as about 20-25 years of age, when most auxological aspects have been reached, and sexual maturity completed. Consequently, I believe that each person should start a "health diary" at that age. Therefore, also healthy people should be monitored as well as patients, which should be one of the tenets of preventive medicine.

Having said that, I must now say that chronological age is practically meaningless in calculating health status. In fact, only a very careful analysis of an individual's personal functional and morphological aspects will reveal early signs of disease, and enable physicians to prevent its progression. This, of course, applies much more to multimorbidity; in fact, once identified them, measures can be made to eradicate or to delay the start or the progression of each illness, therefore determining a better state of health during the progression of chronological age.

All this would revolutionize today's medicine where the physician looks for early signs and symptoms of each possible alteration/disease, before deciding on treatment. The revolution I am talking about is to look at each individual when they are enjoying still normal health, as mentioned above. Indeed, current practice will become to measure a large series of functional laboratory parameters relating to all the functional assets of single tissues and/or organs, and also by imaging assessment, namely by looking at the morphological aspects of all the organs and tissues of the human body, mainly using echography approaches, and also by making a total body scan of different parts of the organism, and this, as said before, starting very early, i.e., at 20-25 years old.

This approach may be considered too costly, but in effect it is much less costly than waiting for the appearance of an overt disease, which must then be treated for decades, frequently with very expensive drugs and tests (laboratory and imaging). In this short presentation, I'll also discuss theoretical and practical methods that can be used in the practice of predictive medicine at genomic level, as well as secondary preventive aspects aimed at improving the health of each individual, taking into consideration the primary prevention of diseases, also by decreasing environmental nocive factors, and tailor beneficial personal life style approaches for each person.

The inversion of the "paradigm" between aging and disease (1), the misnomers of the use of the term "age-associated disease", and other considerations related to ideological aspects of so-called oxymoron term, physiological aging, are discussed to illustrate the need for this expected revolution in medical care, including Laboratory Medicine approaches. This will also support the joining of Preventive Medicine to effective Individualized Medicine.

\section{Reference}

1. Salvatore F. The shift of the paradigm between ageing and diseases. Clin Chem.Lab Med. 2020 Sep 25;58(10):1635-1644. https://doi.org/10.1515/ cclm-2020-0125. PMID:32286241.

\section{TESTING FOR SARS-COV-2: SELF-SAMPLING AND PRE-ANALYTICAL ISSUES}

\section{A. Padoan}

Department of Medicine, DIMED, University of Padova and Department of Laboratory Medicine, University Hospital of Padova, Italy

Although reverse real-time PCR (rRT-PCR) remains the gold standard for detecting SARS-CoV-2, high tests demanding has overwhelmed molecular laboratory capacities in all countries around the world, especially during early pandemics. 
During the second wave, the validation of SARS-CoV-2 antigen rapid diagnostic tests (RDT) has substantially changed testing strategies globally, since results were available within $30 \mathrm{~min}$, reducing turnaround time and therefore exposure risk. Recently, validated self-tests for SARS-CoV-2 based on the nasopharyngeal swab (NPS) or saliva have prompted for the empowerment of the general population in the fight against the spread of infectious.

Swabbing is a complex task requiring training and competency assessment, and thus they are performed by trained nurses or physicians. The complexity of NPS, coupled with a lack of a standard swabbing practice may contribute to a high number of false-negative results for SARS-CoV-2. SARS-CoV-2 rRT-PCR false-negative results have been reported to be as high as $41 \%$ and several reports exist of patients negative to NPS, who are subsequently positive on repeat testing [1]. Differently, the false-positive ratio for the SARS-CoV-2 molecular test is expected to be very low, since PCR design is mostly unaffected by false-positive results. Recently, Tsang et al. compared the diagnostic performance of different clinical specimens, including nasopharyngeal, nasal, throat, and oropharyngeal swabs and saliva and they found that using NPS as the gold standard, moderate sensitivities were achieved by saliva $(85 \%, 75-93)$ and nasal swabs $(86 \%, 77-93)$ and a much lower sensitivity by throat swabs (68\%, 35-94). The Authors concluded that saliva and nasal swabs are clinically acceptable alternatives to commonly used nasopharyngeal swabs. Saliva is a matrix elective for self-collection, and molecular testing is reliable but require laboratory instrumentation to be performed. Indeed, antigen determination on salivary samples is still under debate [2].

Most of the errors occur in the preanalytical phase, with relatively few analytical and post-analytical errors. Some issues arising during the pre-analytical phase of SARS-CoV-2 diagnostics regards: the time of swab, swabbing practice, sample handling and conservation and RNA extraction. NPS should be taken at the time of symptom onset when the highest viral load occurs in COVID-19, thus not the day immediately before (and not too far from) possible close contact with positive subjects. Sample handling and storage were only partially a limiting factor when samples are kept a $4^{\circ} \mathrm{C}$ and processed within 5 days [2]. Differently, sample preparation is a crucial factor for antigen testing, and centrifuged vs. non-centrifuged samples give discordant results.

In conclusion, self-testing could be of aid in the screening programs for reducing viral spread, but other alternatives are possible, such as self-collection of samples with analytical tests performed in clinical laboratories. These required the optimization of pre-analytical steps to reduce the impact on results.

\title{
References
}

1. Woloshin, S.; Patel, N.; Kesselheim, A.S. False Negative Tests for SARS-CoV-2 Infection - Challenges and Implications. N. Engl. J. Med. 2020, 383, e38.

2. Basso, D.; Aita, A.; Navaglia, F.; Franchin, E.; Fioretto, P.; Moz, S.; Bozzato, D.; Zambon, C.-F.; Martin, B.; Dal Prà, C.; et al. SARS-CoV-2 RNA identification in nasopharyngeal swabs: issues in pre-analytics. Clin. Chem. Lab. Med. 2020, 58, 1579-1586.

\section{NEW ORGANIZATIONAL MODEL FOR DECENTRALIZED MANAGEMENT OF ORAL ANTICOAGULANT THERAPY OF ULSS 6 EUGANEA}

\author{
E. Gnatta, R. Ghirardo, V. Temporin, A.M. Leo \\ UOC Medicina di Laboratorio Ospedali Riuniti Padova Sud “Madre Teresa di Calcutta” Monselice (Padova-Italy)
}

Background: Point of Care (POCT) portable coagulometers allow to shift determination of prothrombin time (PT-INR) from central laboratory to peripheral health facilities. In a broad and complex territory such as that of ULSS 6 Euganea, composed of five local health districts with a population of about 936.000 inhabitants, determining PT-INR in territorial decentralized offices is a key objective in therapy control and patient's life quality improvement. Aim of the study: description of the organizational model for decentralized management of patients in Oral Anticoagulant Therapy (OAT) involving Anticoagulation Clinic (AC), Integrated Home Cares (IHCs) and Routine Medical Cares (RMCs). Methods: Starting from October 2020, a bidirectional connection was implemented between Laboratory Information System (LIS) of the Ospedali Riuniti Padova Sud "Madre Teresa di Calcutta" Monselice (Padova, Italy) and peripheral offices, where 
clinical data are collected and PT-INR is determined by POCT portable coagulometers (Coaguchek ${ }^{\circledR}$ Roche Diagnostics, Germany). Information is sent in real time to central laboratory using IT1000 middleware, where data are validated and historicized. Historicized data can be consulted and downloaded like other laboratory exams. Furthermore, for patients followed by AC, data are sent to OAT monitoring software (GESTAOWEB ${ }^{\circledR}$ Tesigroup Milan). Results: the project involved 2980 patients. Centres to which patients are belonging are located throughout the territory of ULSS 6 Euganea and consist of 7 IHCs, 9 RMCs and one AC, a total of 81 POCT instruments for about 60.000 determinations per year. From January 1st 2021 to June 30th 2021 the average frequency of determinations per patient was about 30 days, while the average number of determinations per patient was about 7,5. Conclusions: the connection with LIS represents project's focal point, allowing historicization of data, making them available at any time for any connected facility. This model simplifies management of both patients in IHC and followed by RMC, allowing easier access to the determination of PT-INR, with more constant therapy control and significant improvement of life quality.

\section{THE ROLE OF PRESCRIBERS: THE PDTA IN OVARIAN CANCERS}

\section{P. Scollo}

Direttore del Dipartimento Materno Infantile e dell'U.O.C. di Ginecologia ed Ostetricia dell'Azienda Ospedaliera per l’Emergenza Cannizzaro (A.O.E.C.) di Catania

Ovarian cancer is the seventh most frequent malignancy in the female population worldwide and the leading cause of death among gynecological cancers. In Italy, about 5300 new cases were registered in 2019. It is the fifth cause of death for cancer in women aged 50 to 69 , with a 5 years survival from diagnosis estimated at $40 \%$.

Nowadays, there is a lack of effective screening strategies for the early identification of the disease, and the absence of predictive symptoms and the resulting delay in diagnosis means that more than $75 \%$ of women present advanced cancer at the time of diagnosis, requiring a multidisciplinary management by professionals with specific skills.

Only referenced centers can offer such complex assistance and it is well known how patient survival curves improve $(>40 \%)$ in centers with specific experience in the treatment of ovarian cancer.

The availability of a guide in the diagnostic paths is a requirement for general practitioners and specialists of other disciplines that arises from the need to guarantee the most appropriate, less demanding, more useful and less expensive diagnostic path. The purpose of PDTAs is to increase the quality of perceived and effectively delivered care, improving outcomes and promoting patient safety through the use of the right resources needed.

The surgical approach plays a fundamental and essential role in the treatment program and the absent tumor residue has been defined as the only tumor residue associated with optimal survival curves and also the antiblastic chemotherapy, that always follows surgery in advanced ovarian cancer, gets better results after optimal surgery. The operator's experience, culture and technical preparation have an impact on the patient's survival curve, which is why it is essential that this procedure be performed in specialized reference centers, with an experience of the gynecologist-oncologist surgeon certified by an adequate training course, case history and an overall multidisciplinary structural organization, in order to guarantee the best therapeutic sequence for the patient. The first network of reference and dissemination of PDTAs is constituted by general medicine.

Indeed, we cannot ignore sharing with the entire regional network of general practitioner, who will thus know who to send the patient with suspicion or already ascertained diagnosis of ovarian cancer, for an adequate diagnostic and therapeutic path, allowing feedback on the conditions and clinical-therapeutic pathways for individual patients. In addition, information and reference relationships with voluntary associations and patient associations must be encouraged. They constitute a fundamental asset in the creation and development of information, health culture of primary and secondary prevention and support in home care or in assistance facilities even to the terminally ill. 


\title{
THE ORAL ANTICOAGULANT THERAPY CLINICAL MANAGEMENT DURING PANDEMIA: THE SOLUTION FOUND BY THE FCSA 123 HEMOSTASIS AND THROMBOSIS CENTER IN SAVONA
}

\author{
C. Scarone, V. Dovere, C. Traverso, F. Lillo \\ S.C. Laboratorio di Patologia Clinica ASL 2 Regione Liguria
}

Background: The emergency caused by the Covid-19 pandemic has forced the reformulation of the operating methods of the Health System, turning the spotlight on the need for greater interaction between hospital and territory. This aspect is even more evident in patients needing for a more strict followup as those under antithrombotic therapy (TAO), making clear the essential usefulness of digital tools and of new organizative models. Objectives: Minimize the risks for adverse events in patients on oral anticoagulant therapy with vitamin K inhibitor drugs (AVK) or direct oral anticoagulants (DOAC), maintaining an optimal level of clinical/consulting support, allowing accurate monitoring of compliance and managing both hemorrhagic and thrombotic emergencies remotely, in order to reduce access to the Emergency Room (when possible) and to hospital facilities. Methods: The patients in charge at the Thrombosis Center of the San Paolo Hospital in Savona (FCSA 123) are in total 781: 66\% diagnosed with atrial fibrillation (FA), $20 \%$ for the presence of mechanical or biological valve prostheses and $14 \%$ for thrombotic pathologies such as deep vein thrombosis (DV) and pulmonary embolisms (EP). 560 patients are in DOAC, while 221 patients in AVK. During the pandemic, a dedicated mobile telephone number was activated (12 hours/day from $8 \mathrm{am}$ to $8 \mathrm{pm}$ ) equipped with an instant messaging application service. Each patient was asked for an email address and signed consent to the computerized management of TAO therapy and forwarding of health documents. Results: In order to monitor the expected results, the following indicators were evaluated: number of incoming phone calls; number of treatment plans issued for DOAC; time in range for patients in AVK; number of complications recorded in the period under review (April 2020-April 2021). The results obtained are respectively: about 9000 phone calls received; 560 treatment plans released; time in range from patients in AVK (TTR) 74\%; 22 adverse events including two major hemorrhages. Conclusions: The new organization, based on digital support of clinical monitoring, has received high appreciation from patients and consequently a greater compliance with the therapy protocol. This management model has allowed an effective control both of the number and severity of adverse events, while the reduction of outpatient access has allowed to drastically reduce the infectious risk. In addition, e-mailing of reports and treatment plans allowed an optimization of human resources.

\section{HEMATOLOGICAL DIAGNOSTICS: THE TELEMEDICINE PROJECT FOR THE LABORATORY SPREAD ACROSS A LARGE TERRITORY}

\author{
M. Lorubbio $^{1}$, F. Baldelli ${ }^{1}$, E. Bromo ${ }^{5}$, G.P. Caldarelli ${ }^{4}$, C. Donnini ${ }^{1}$, S. Fabbroni ${ }^{1}$, A. Fanelli ${ }^{1}$, M. Fantacci ${ }^{2}$, L. \\ Gasbarri $^{1}$, M. Mazzi ${ }^{4}$, A. Periccioli ${ }^{3}$, P. Pugliano ${ }^{4}$, C. Rapini ${ }^{2}$, A. Rebuffat ${ }^{3}$, A. Sereni ${ }^{1}$, M. Sorini ${ }^{1}$, E. Tripodo ${ }^{1}$, A. \\ Ognibene $^{1}$
}

${ }^{1}$ Clinical Chemical Analysis Laboratory, San Donato Arezzo Hospital

${ }^{2}$ Clinical Chemical Analysis Laboratory, Montepulciano Valdichiana Reunited Hospital

${ }^{3}$ Clinical Chemical Analysis Laboratory, Hospital Alta Valdelsa

${ }^{4}$ Clinical Chemical Analysis Laboratory, Misericordia Grosseto Hospital

${ }^{5}$ Dip. del Farmaco, Misericordia Grosseto Hospital

Introduction. The territory of the USL TUSCANY SOUTH EAST (TSE) has an area of 11,557 sq km with 850,446 inhabitants. The use of information and communication technologies (ICT) and E-Health can contribute to a reorganization by moving the focus of health care from the hospital to the territory. The aim of the present project is to evaluate of the complete blood count (CBC) test and the peripheral blood smear through digital images, shared and available to the team of the 12 TSE laboratories. During implementation, the image analyzers and the staining adopted were compared, together with the quality indicators (QI) to support the new flows implemented between the peripheral laboratories and the Hub laboratory. Materials and methods The connection network between all TSE Laboratories was guaranteed by the DMS (DASIT Management System) WEB application, for XN (Sysmex) 
instruments and, Caresphere TMXQC for the management of CQI. The cellular digital images were shared through digital online archives, managed using the DI60 and DC-1 (CellaVision ${ }^{\mathrm{TM}}$ ). 135 peripheral blood smears were performed using SP10 (HUB) and HemaPrep (Spoke), staining May-Grünwald Giemsa (Sysmex) and Romanowsky-type (RAL Diagnostics), respectively. For study QI, 26 CBC performed in the peripheral laboratories were reanalyzed after 4-6 $\mathrm{h}$ at the Arezzo Laboratory. Statistical analysis was performed using SPSS and GraphPad Prism. Results. The Passing-Bablok and Bland-Altman plot analysis performed for comparison of all elements of the blood count test, provided excellent results between the technologies and the different cell staining used (data not shown). The MCV and PLT parameters of the $\mathrm{CBC}$, performed in the spoke laboratories and re-performed in the Hub laboratory after 4$7 \mathrm{~h}$, showed a statistical significative differences of $3.18( \pm 0.66) \mathrm{fL}$ and $10.69( \pm 17.83) 103 \mu \mathrm{L}$ respectively. Conclusions. The organization proposed in the project improves the analytical quality, harmonises the reporting and interpretation of analytical data, promotes uniform training, preparing for continuous professional comparison. This approach represents a model that contrasts with centralization, favoring the concept of widespread laboratory medicine, while maintaining an advantageous cost/benefit ratio.

\title{
LEUCOCYTE DIFFERENTIAL AND MORPHOMETRIC PARAMETERS WITH MINDRAY BC-6800 PLUS: A POSSIBLE PREDICTIVE TOOL TO DIAGNOSE SEPSIS AND SARS-COV-2 INFECTIONS
}

\author{
$\underline{\text { M. Pelagalli }}^{1,2}$, A. Giovannelli ${ }^{1,2}$, C. Calabrese ${ }^{1,2}$, S. Sarubbi ${ }^{1,2}$, M. Minieri $^{1,2}$, M. Nuccetelli ${ }^{1,2}$, M. Pieri $^{1,2}$, S. Bernardini $^{1,2}$ \\ ${ }^{1}$ Department of Laboratory Medicine, “Tor Vergata” University Hospital, Rome, Italy \\ ${ }^{2}$ Department of Experimental Medicine, University of "Tor Vergata", Rome, Italy
}

Background. Sepsis is an infectious disease (the etiology can be viral or bacterial) with hight mortality, threatening human health. Clinicians need to diagnose the patient's infection in time and look for pathogens in order to develop an effective treatment plan; therefore, a quickly and early screen to diagnose sepsis has become an urgent problem in clinical laboratories. Different inflammatory factors are used to diagnose the sepsis; CRP, IL-6, PCT, ADM, lactate, D-dimer etc., but they also have limitations such as insufficient sensitivity and specificity and requiring additional examination cost. The aim of this study is to use leucocyte counts (neutrophils and monocytes that are activated from pathogenic virus or bacteria) and others morphological change with Mindray BC-6800-plus platform to diagnose sepsis early, quickly, conveniently and at low cost. Methods. A total 957 EDTA-k2 anticoagulant venous whole blood samples were collected: 70 control patients (blood donors) with a normal complete count blood and negative VES, and 887 samples hospitalized at the emergency department with symptoms attributable to sepsis with PCT request. All data was divided in 4 groups: control group, group where sepsis cannot be confirmed, group with confirmed sepsis diagnosis and a group with sepsis from SARS-CoV-2 infection. Morphometric and numeric parameters are reported with Mindray BC-6800 plus: blood count like positional parameters X, Y, Z of neutrophils, lymphocites and monocytes, PLT, NLR (neutrophil lymphocyte ratio) and IMG (index of immature granulocytes). For statistical analysis was used Shapiro Wilk test for distribution analysis and the non parametric Kruskal Wallis test to evaluate significative differences among the groups $(p<0.05)$ and also examined ROC curve analysis. Results. There is a statistically significant difference between control group and sepsis group for haematological parameters: positional parameters (Neu X, Y, Z; Mon X, Y, Z and Lym X, Y, Z), IMG, NLR, PLT. The roc curves highlight acceptable sensitivity and specificity values for some haematological parameters and suggest a possible cut-off. Conclusions. The BC-6800 plus can help the diagnosis of sepsis upon the admission to the emergency department using some morphological positional parameters.

\section{THE BIG DATA ANALYSIS APPLICATION TO EVALUATE THE CLINICAL UTILITY OF IGM ANTI SARS-COV-2 DETERMINATION: THE EXPERIENCE OF MODENA}

\author{
V. Pecoraro ${ }^{1}$, T. Pirotti $^{1}$, T. Trenti ${ }^{1}$, M. Plebani ${ }^{2}$ \\ ${ }^{1}$ Department of Laboratory Medicine and Pathology, Azienda USL of Modena, Italy
}


${ }^{2}$ Department of Laboratory Medicine, University-Hospital of Padova, Italy.

Background. Serological tests identifying SARS-CoV-2 IgG and IgM in serum play an important role in understanding the disease epidemiology. However, their immunological significance are currently undefined. There are many methods available for the detection of specific Abs whit suboptimal diagnostic accuracy and relatively high throughput capacity and less stringent specimen requirements compared to RNA-based assays. We aimed to assess the clinical utility of IgM detection in SARS-CoV-2 using the big data analysis. Methods. We conduct a retrospective study analyzing with a big data analysis all samples collected between 11 March and 30 September 2020. All serum samples received at the laboratory were processed using qualitative and commercially available rapid lateral flow immunoassay tests for 2019- nCoV IgG and IgM. Positive results were confirmed using a chemiluminescent method. Subjects with a positive result were contacted from the Department of Public Health for further tests (viral RNA research or subsequent serological tests) for definitive diagnosis. Results. A total of 69,343 serological tests (in 42,911 subjects) and 140,065 oropharyngeal or nasopharyngeal swabs (in 88,771 subjects) were performed. $94.5 \%$ of subjects screened $(n=40,559)$ had negative results for both IgG and IgM. Of the 640 subjects with both IgG and IgM positive results, viral RNA research confirmed positivity in $16 \%$. Of the subjects with IgG negative and IgM positive results, a positivity was confirmed in $1.4 \%$ ( $\mathrm{n}=7 / 478)$ subjects. Subsequent serological testing confirmed IgG positivity in 8 subjects (1.6\%). Conversely, in subjects with IgG positive and IgM negative results, a positivity was confirmed in $7.9 \%$. Therefore, analysis suggests that up to $94 \%$ of serological test results of IgM positivity and IgG negativity are false positive whereas, serological test results of IgG positive and IgM negative are confirmed true positives in around $7.9 \%$ of subjects. Discussion. Our study, based on big data analysis application, confirms the scarce clinical utility of IgM anti SARS-CoV-2 detection in COVID-19 management, and underlines the responsibility of laboratory medicine professionals to highlight limitations of the SARS-CoV-2 serological tests due to uncertainty in their interpretation.

\title{
LARGE GENOMIC ALTERATIONS (LGAS) PROFILES IN HBOC PATIENTS USING SHALLOW WGS (SWGS) PIPELINE FOR THE ASSESSMENT OF HOMOLOGOUS RECOMBINATION DEFICIENCY (HRD) SCORE
}

\section{G.L. Scaglione, C. Nardelli, M. Setaro, E.D. Capoluongo}

\author{
Lab. Genomica Oncologica, CEINGE Biotecnologie Avanzate S.C.R.L., Napoli
}

Background. The homologous recombination (HR) pathway is essential for DNA double strand break (DSB) repair and involves several genes. HR deficiency (HRD) arises upon inactivation of BRCA1/2, RAD51C or PALB2. The impairment of this pathway is a common characteristic of many tumors and it is frequently observed in breast and ovarian cancer. Consequently, accurate detection of HRD is of clinical relevance as it is indicative of sensitivity to targeted therapy with poly ADP-ribose polymerase inhibitors (PARPi) as well as to DNA damaging reagents. Methods Shallow Whole Genome Sequencing (sWGS) was performed on 16 ovarian cancer (OC) samples. The training set included 13 samples ( 6 somatic, 7 germline) carrying both $\mathrm{BRCA}+\mathrm{ve}(\mathrm{n}=9)$ and BRCA-ve $(\mathrm{n}=4)$ status. The germline and somatic samples were prepared according to SeqCap EZ HyperCap protocol (Roche). Briefly, $50 \mathrm{ng}$ of input DNA was used for libraries preparation using the KAPA HyperPlus Library Preparation Kit (Roche). Samples were then pooled and sequenced on NextSeq550 Dx platform (Illumina). Sequencing files were quality checked, analyzed and processed using our dedicated bioinformatics pipeline. In this workflow, LGAs profiles were calculated using whole genome sequencing data at low coverage (0.41.0X) using different sliding window size spanning 5 to $1000 \mathrm{Kbases}$. The HRD score was then estimated by measuring the level of agreement in the segmentation profiles of each samples. Results. The BRCA status was assessed in 13 samples (training set) and the HRD score was estimated as follow: 2 of the 3 somatic BRCA positive (sBRCA+ve) samples were classified as HRD positive while 2 out of 3 sBRCA-ve samples were scored as negative. As expected, both positive (+ve) and negative (-ve) germline BRCA (gBRCA) samples were classified as HRD negative. Lastly, Among the remaining samples $(n=3)$ for which the germline status of BRCA genes was not available, the algorithm classified 1 sample carrying PALB2 $\mathrm{CNV}$ as HRD positive. Conclusions. Germline BRCA1/2 mutation status is currently the main genetic biomarker of HRD but it has its drawbacks: among others HRD can be driven purely by somatic events. In this scenario we have customized a 
simple and robust bioinformatics workflow to infer the HR status of breast and ovarian tumor based on sWGS to support patients' treatments and follow up strategies.

\title{
DETERMINATION OF SARS-COV-2 ANTIBODIES IN SALIVARY SAMPLES FROM VACCINATED INDIVIDUALS AND COVID-19 PATIENTS
}

\author{
C. Cosma ${ }^{1}$ A. Padoan $^{1}$, C. Di Chiara ${ }^{2}$, D. Rinaldi ${ }^{1}$, D. Donà ${ }^{2}$, A. Gastaldi ${ }^{3}$, D. Basso ${ }^{1}$, C. Giaquinto ${ }^{2}$, M. Plebani ${ }^{1}$ \\ ${ }^{1}$ Department of Laboratory Medicine, University-Hospital of Padua, Italy \\ 2Division of Pediatric Infectious Diseases, Department for Women's and Children's Health, University of Padua, Italy \\ ${ }^{3}$ Department of Pediatrics, Woman and Child Hospital, University of Verona, Verona, Italy
}

Background and Aim: Salivary SARS-CoV-2 Ab determination could be suitable for monitoring the viral spread and vaccination efficacy, especially in pediatric patients. We investigated N/S1-RBD IgG antibody levels in salivary samples of infectious-naïve vaccinated subjects and of COVID-19 patients, further comparing levels with serum anti-SARS-CoV-2 S-RBD IgG. Methods: A total of 72 subjects were enrolled at the Padova University Hospital: 36 COVID-19 patients and 36 health care workers (HCW), who underwent a complete vaccination campaign with BNT162b2 (BioNTech/Pfizer). All collected a salivary sample, using Salivette (Sarstedt, Nümbrecht Germany). For 9 HCW, salivary samples were collected at three different times within the same day (before breakfast, at $10 \mathrm{am}$, and after lunch). A serum sample was also collected for all individuals. Time post symptoms onset or time from the first vaccine were also recorded. Salivary COVID-19 N/S1 RBD (sal-IgG) ELISA (RayBiotech, GA, USA) and anti-SARS-CoV-2 S-RBD IgG Ab (ser-IgG) (Snibe Diagnostics, Shenzhen, China) were used for determining IgG Ab. Results: Subjects' mean age ( \pm sd) was $35.8 \pm 18.2$ yrs. Age significantly differed $(\mathrm{p}<0.001)$. Salivary IgG levels were not influenced by time post-symptom onset or time postvaccination, both on vaccinated HCW (rho=-0.147, $\mathrm{p}=0.402$ ) and COVID-19 subjects ( $r h o=0.0267, p=0.986$ ). Ser-IgG levels was not influenced by the time post-symptom onset for COVID-19 subjects ( $\mathrm{rho}=0.102, \mathrm{p}=0.419$ ), while a strong significant correlation was found with time post-vaccination in HCW ( $\mathrm{rho}=-0.6292, \mathrm{p}<0.001)$. Sal-IgG levels were notinfluenced by the daytime of collection ( $\mathrm{rho}=0.148, \mathrm{p}=0.373$ ). Passing-Bablok regressions showed that sar-IgG and serIgG comparability was assessable only when ser-IgG values were divided by 1000, being slope and intercept $0.068(95 \%$ CI: $0.069-0.341$ ) and 0.221 (95\% CI: -0.097 to 0.786), respectively. Conclusions: Salivary IgG is efficiently detectable both in COVID-19 and in vaccinated individuals and analyses appeared to be not influenced by the daytime of collection. The analyses performed showed that, overall, sal-IgG were lower than ser-IgG, and thus comparability with serum levels needs to be better explored.

\section{MDW IS A NOVEL INFLAMMATORY BIOMARKER WITH PROGNOSTIC RELEVANCE IN COVID-19 PATIENTS}

\author{
G. Riva ${ }^{1}$, V. Nasillo ${ }^{1}$, A.M. Ottomano1, G. Bergonzini ${ }^{1}$, A. Paolini ${ }^{2}$, B. Lusenti ${ }^{1}$, S. Castellano ${ }^{1}$, R. Rizkallah ${ }^{1}$, \\ P. Ferrari ${ }^{1}$, M. Varani ${ }^{1}$, M. Luppi ${ }^{2}$, E. Tagliafico ${ }^{1}$, T. Trenti ${ }^{1}$ \\ ${ }^{1}$ Dip. Int. Interaz. Medicina di Laboratorio e Anatomia Patologica, AOU/AUSL di Modena, Italy \\ ${ }^{2}$ U.O. Ematologia, Univ. Modena e Reggio Emilia, AOU di Modena, Italy
}

Monocyte Distribution Width (MDW), a new hematologic parameter correlating with cytomorphologic changes occurring during monocyte activation, has recently been described as promising early biomarker of sepsis. Similar to sepsis, in SARS-CoV-2-associated disease (COVID-19), monocyte/macrophage subsets are considered key mediators of the lifethreatening hyperinflammatory disorder -commonly defined as 'cytokine storm'- which is part of the complex infectionassociated immune dysregulation observed in severe COVID-19 cases (possibly, representing a new kind of viral sepsis). Thus, we aimed at investigating possible prognostic roles of MDW testing during monitoring of COVID-19 patients. In this work, we longitudinally measured MDW values in a cohort of 87 patients with molecularly-proven COVID-19 diagnosis, consecutively admitted to our intensive/subintensive clinics in early 2020. Statistical analyses were applied to correlate 
MDW values with common inflammatory markers, disease severity, clinical trajectories and final outcome. We found significant direct correlations between MDW and different inflammatory markers routinely assessed during hospitalization, namely CRP ( $\mathrm{p}<0.001)$, fibrinogen $(\mathrm{p}<0.001)$ and ferritin $(\mathrm{p}<0.01)$. Moreover, high MDW values were remarkably associated with fatal outcome (AUC=0.76, sensitivity 0.75 , specificity 0.70, MDW threshold 26.4; $R R=4.91, O R=7.14$ ). Furthermore, evaluating MDW dynamics in cases with longer follow-up, we frequently observed progressive MDW increments in patients with worsening inflammatory conditions, while clinical recoveries were consistently associated with MDW decreases. Our study shows, for the first time, that MDW can be useful in the prognostic monitoring of hospitalized COVID-19 patients, as it is: (i) easy and rapid to obtain, (ii) directly related to the activation state of a fundamental inflammatory cell subset (i.e. monocytes, pivotal both in cytokine storm and in sepsis immunopathogenesis), (iii) strongly correlated with clinical severity of COVID-19-associated inflammatory disorder, and, in turn, (iv) endowed with relevant prognostic significance. Additional studies are needed to define the role of MDW monitoring in other clinical settings, including COVID-19 outpatients.

\title{
MELANOQ WEB-APP: AN INNOVATIVE TOOL FOR COLLECTING, HARMONIZING, AND MANAGING CLINICAL AND GENETIC DATA OF MELANOMA PATIENTS USING A WEB-BASED PLATFORM
}

\author{
C. Pellegrini, L. Cardelli, M. Mastrangelo, M. Bressan, M.C. Fargnoli
}

Dermatologia, Dipartimento di Scienze Cliniche Applicate e Biotecnologiche, Università degli Studi dell’Aquila

With more than 320,000 new cases and 57,000 deaths worldwide in 2020, melanoma remains one of most aggressive cancer globally. The incidence varies widely among white-skinned populations and has significantly increased in recent years in Australia, US and in Europe, including in the Mediterranean area. Given the heterogeneous recording of epidemiological, clinical and genetic variables across melanoma studies, the MelaNostrum Consortium was confronted with the difficult task of harmonizing all information from different international centers to perform association studies. Therefore, MelaNostrum investigators developed a questionnaire for data collection that could be used as a full or itemized template for the standardization. On the base of that questionnaire, we built a web-based tool that allow to collect data taking advantage of a telemedicine approach. The App allows to complete online the MelanoQ questionnaire using a tablet, and automatically generates the database with all the collected data of each patient. Similar to the MelanoQ questionnaire, the MelanoQ Web-App is organized in 4 main sections (A-D) and includes a total number of 64 items related to: general and demographic information (section A); phenotypic, UV exposure risk factors and lifestyle habits (section B); clinical examination, medical history and family history (section C); tumor characteristics, including histology, staging and molecular profile (section D). Different subsections are designed for self-administration, patient/ control interviews performed by a physician or study nurse, and data collection from medical records. A specific attention has been paid to the anonymization of data, to solve privacy concerns and to collect different signed consensus format by international patients.In conclusion, we sought that a web-tool to generate a comprehensive database allowing to pool data on melanoma from different centers world-wide is of great opportunity for clinicians and for patients.

\section{EVALUATION OF A HIGH-SENSITIVITY CARDIAC TROPONIN POINT-OF-CARE ASSAY: A COMPARISON WITH A LABORATORY PLATFORM}

\author{
$\underline{\text { R. Aloe }}^{1}$, P. Avanzini ${ }^{1}$, R. Musa ${ }^{1}$, P. Cerati ${ }^{1}$, M. Di Pietro ${ }^{2}$ \\ ${ }^{1}$ SSD Biochimica ad Elevata Automazione, AOU Parma \\ ${ }^{2}$ Laboratorio Unico Metropolitano (LUM), AUSL Bologna
}

Background: Until recently, high sensitivity in cardiac troponin testing was achieved exclusively by laboratory platforms, whereas point-of-care systems lacked the required analytical performance. In 2020, however, the first high sensitivity cTn POC assay (TriageTrue, Quidel) was successfully evaluated [1] and assay-specific cut-offs for the 0/1-h algorithm were 
included in the ESC guidelines for the management of NSTEMI [2]. In this work, the performance of TriageTrue in comparison with the hs-cTn assay currently in use in our institution (Access Hs TnI, Beckman Coulter) was evaluated. Methods: Linearity of TriageTrue was assessed on 11 plasma samples, each in 5 replicates (cTn=3.13-800 ng/L). Precision was evaluated on 3 pools with different concentrations ( 5 aliquots/pool $\times 5$ days). For the comparison study, 146 plasma T0/T1 samples coming from 73 ED patients with chest pain were tested using both methods. Agreement was assessed with Spearman's correlation, Passing-Bablok regression and Bland-Altman analysis (MedCalc 18.6).

Results: Linearity: Spearman's correlation coefficient=1.000; $<<0.001$. Total precision (CV): 4.5\% (low), 9.2\% (medium), 9.0\% (high). Within-run precision: 4.82-9.25\%. Passing-Bablok regression: intercept=0.6674 (95\% CI: $-0.6031-1.2862)$, slope $=0.886$ (95\% CI: 0.8301-0.9792) for T0; intercept=0.01802 (95\% CI: $-1.0898-0.07439)$, slope=0.9463 (95\% CI: 0.8777-1.0347) for T1. Bland-Altman analysis: mean bias = -0.4 ng/L (T0); $1.4 \mathrm{ng} / \mathrm{L}$ (T1). Spearman's correlation: 0.914 (95\% CI: 0.865-0.946; p<0.001) for T0; 0.924 (95\% CI: 0.880-0.952; p<0.001) for T1.

Conclusion: CV was $<10 \%$ for all the analyzed pools. Precision was particularly high $(\mathrm{CV}=4.5 \%)$ in the low pool, which is relevant for the reduction of analytical noise and the reporting of small cTn variations around the 99th percentile URL. The tested methods show great concordance, with high correlation for T0/T1 samples and a slight proportional difference at T0. Overall, TriageTrue is a valid alternative to the tested laboratory assay and its adoption by EDs may considerably reduce turnaround times and streamline clinical decision-making at the onset of myocardial infarction.

\section{References}

1. Boeddinghaus et al., JACC $2020,75,1111-1124$

2. https://doi.org/10.1093/eurheartj/ehaa575 